Editorial

\title{
Stability and Bifurcation Analysis of Discrete Dynamical Systems 2020
}

\author{
Abdul Qadeer Khan $\mathbb{D}^{1},{ }^{1}$ Tarek F. Ibrahim $\mathbb{D}^{2,3}$ and Abdul Khaliq $\mathbb{D}^{4}$ \\ ${ }^{1}$ Department of Mathematics, University of Azad Jammu and Kashmir, Muzaffarabad 13100, Pakistan \\ ${ }^{2}$ Department of Mathematics, Faculty of Sciences and Arts (Mahayel), King Khalid University, Abha, Saudi Arabia \\ ${ }^{3}$ Department of Mathematics, Faculty of Science, Mansoura University, Mansoura 35516, Egypt \\ ${ }^{4}$ Department of Mathematics, Riphah Institute of Computing and Applied Sciences, Riphah International University, \\ Lahore Campus, Lahore, Pakistan
}

Correspondence should be addressed to Abdul Qadeer Khan; abdulqadeerkhan1@gmail.com

Received 30 November 2021; Accepted 30 November 2021; Published 15 December 2021

Copyright (C) 2021 Abdul Qadeer Khan et al. This is an open access article distributed under the Creative Commons Attribution License, which permits unrestricted use, distribution, and reproduction in any medium, provided the original work is properly cited.

The importance of difference equations cannot be overemphasized. These equations model discrete physical phenomena on the one hand and are the integral part of numerical schemes used to solve differential equations, on the other hand. This widens the applicability of such equations to many branches of scientific knowledge. Discrete dynamical systems are described by difference equations and potentially have applications in many branches of applied sciences.

This special issue provides a platform to disseminate original research in the fields of discrete dynamical systems and bifurcation theory. This was an excellent opportunity for researchers to share their findings with the scientific community. All manuscripts submitted to this special issue have been reviewed through peer-reviewing process. Based on the reviewers' reports, 11 original research articles have been accepted for publication in this well-reputed journal. A brief summary of each published article in this special issue by providing a short editorial note has also been presented as follows.

In the paper "Asymptotic Stability of Neutral Set-Valued Functional Differential Equation by Fixed Point Method," Bao and Wang have investigated a class of neutral set-valued functional differential equations and obtained a criterion for the globally asymptotic stability theorem with necessary and sufficient conditions by the fixed point method. Finally, Bao and Wang verified the validity of the result by an example.

In the research article "Global Dynamics of Delayed Sigmoid Beverton-Holt Equation," Khyat and Kulenovic investigated certain dynamic scenarios for general competitive maps in the plane and applied to some cases of second-order difference equation $x_{n+1}=f\left(x_{n}, x_{n-1}\right)$, $n=0,1, \ldots$, where $f$ is decreasing in the variable $x_{n}$ and increasing in the variable $x_{n-1}$. Further as a case study, Khyat and Kulenovic used the difference equation $x_{n+1}=x_{n-1}^{2} /$ $c x_{n-1}^{2}+d x_{n}+f, n=0,1, \ldots$, where the initial conditions $x_{-1}, x_{0} \geq 0$ and the parameters satisfy $c, d, f>0$. In this special case, the authors characterized completely the global dynamics of this equation by finding the basins of attraction of its equilibria and periodic solutions. Khyat and Kulenovic also described the global dynamics as a sequence of global transcritical or period-doubling bifurcations.

In the research article "Dynamic Analysis and Chaos Control of Bertrand Triopoly Based on Differentiated Products and Heterogeneous Expectations," Zhao has investigated the dynamic behavior of the Bertrand price game model, the instability of the boundary equilibrium point, and the stability condition of the internal equilibrium point by the stability theory. Furthermore, bifurcation diagram, basin of attraction, and critical curve are introduced to investigate the dynamic behavior of this game. Finally, chaotic behavior of the dynamical system is controlled by time delay feedback control and the numerical analysis showed that the effective control of the dynamical system can be unstable behavior and the rapid recovery of the market can be stable and orderly.

In the article "Global Dynamical Properties of Rational Higher-Order System of Difference Equations," Khan and 
Qureshi have investigated the global dynamical properties of the rational higher-order system. It is explored that under certain parametric conditions, the discrete-time system has at most eight equilibria. By the method of linearization, local dynamics has been explored. Khan and Qureshi explored that positive solution of the system is bounded, and moreover, fixed point $P_{000}$ is globally stable if $\alpha_{1} / \alpha_{2}<1, \alpha_{4} / \alpha_{5}<1, \alpha_{7} / \alpha_{8}<1$. It is also investigated that the positive solution of the system under consideration converges to $P_{000}$. Lastly, theoretical results are confirmed by numerical simulation.

In the article "Periodic Property and Asymptotic Behavior for a Discrete Ratio-Dependent Food-Chain System with Delays," Xu et al. have investigated the discrete ratiodependent food-chain system with delay. By using Gaines and Mawhin's continuation theorem of coincidence degree theory and the method of Lyapunov function, a set of sufficient conditions for the existence of positive periodic solutions and global asymptotic stability of the model are established.

In the original research article "Global Stability for a Semidiscrete Logistic System with Feedback Control," Xu et al. have proposed a semidiscrete logistic model with the Dirichlet boundary conditions and feedback controls. By means of the sub- and supper-solution method and eigenvalue theory, the unique positive equilibrium is proved. By constructing a suitable Lyapunov function, $\mathrm{Xu}$ et al. have investigated the global asymptomatic stability of the unique positive equilibrium. Finally, numerical simulations are presented to verify the effectiveness of the main results.

In the research article "Stability, Neimark-Sacker Bifurcation, and Approximation of the Invariant Curve of Certain Homogeneous Second-Order Fractional Difference Equation," Garic-Demirovic et al. have investigated the local and global character of the unique equilibrium point and boundedness of the solutions of certain homogeneous fractional difference equations with quadratic terms. Moreover, Garic-Demirovic et al. considered the Neimark-Sacker bifurcations and gave the asymptotic approximation of the invariant curve.

In the research article "Lyapunov Stability Analysis of a Delayed Foot-and-Mouth Disease Model with Animal Vaccination," Gashirai et al. have presented a time-delayed foot-and-mouth disease model that incorporates relevant biological and ecological factors, vaccination effects, and disease carriers. Moreover, Gashirai et al. have determined the basic reproduction number and demonstrated that it is an important metric for persistence and extinction of the disease in the community. Finally, numerical illustrations have been given to support some of the analytical results.

In the article "On Stability Analysis of Higher-Order Rational Difference Equation," Khaliq et al. have investigated equilibrium points, local asymptotic stability of equilibrium points, global behavior of equilibrium points, and boundedness and periodicity of the rational recursive sequence $w_{n+1}=w_{n-p}\left(\alpha+\left(\beta w_{n} / \gamma w_{n}+\delta w_{n-r}\right)\right)$, where $w_{n} \neq$ $-\delta w_{n-r}$ for $r \in(0, \infty), \alpha, \beta, \gamma, \delta \in(0, \infty)$, and $r>p \geq 0$. Finally, numerical examples are given to verify theoretical results.
In the article "Existence of Solutions for the Discrete Dirichlet Problem Involving $p$-Mean Curvature Operator," Wang and Zhou have investigated the Dirichlet boundary value problem of the difference equation with $p$-mean curvature operator. Under some determinate growth conditions on the nonlinear term, the existence of one solution or two nontrivial solutions is obtained via variation methods and some analysis techniques. Finally, numerical examples are given to verify theoretical results.

Finally, in the article "Stability and Hopf Bifurcation of a Generalized Chikungunya Virus Infection Model with Two Modes of Transmission and Delays," Besbassi et al. have proposed and investigated a generalized chikungunya virus (CHIKV) infection model with nonlinear incidence functions and two time delays. The proposed model takes into account both modes of transmission that are virus-to-cell infection and cell-to-cell transmission. Furthermore, the local and global stabilities of the disease-free equilibrium and the chronic infection equilibrium have been established by using the linearization and Lyapunov functional methods. Moreover, the existence of Hopf bifurcation is also analyzed. Finally, an application is presented in order to support the analytical results.

\section{Conflicts of Interest}

The editors declare that they have no conflicts of interest regarding the publication of this special issue.

\section{Acknowledgments}

We would like to pay a great homage to all of the authors for their valuable contributions rendered in this respect and also to the reviewers for their valuable suggestions made in the evaluation of the papers during the reviewing process.

Abdul Qadeer Khan Tarek F. Ibrahim Abdul Khaliq 